SLAC-TN-10-037

LCLS-TN-08-6

\title{
Tuning The Laser Heater Undulator
}

\author{
Zachary Wolf \\ SLAC
}

July 24, 2008

\begin{abstract}
The laser heater undulator for the LCLS is a pure permanent magnet device requiring different tuning techniques than the main undulators. In this note, the laser heater undulator is modelled and tuning techniques to compensate various errors are derived.
\end{abstract}

\section{Introduction $^{1}$}

The laser heater undulator for the LCLS requires different tuning techniques than the main undulators. It is a pure permanent magnet (PPM) undulator, rather than the hybrid design of the main undulators. The PPM design allows analytic calculation of the undulator fields. The calculations let errors be introduced and correction techniques be derived. This note describes how the undulator was modelled, and the methods which were found to correct potential errors in the undulator.

\section{Requirements}

The tuning requirements for the laser heater undulator are specified in a Physics Requirements Document. $^{2}$ The tuning requirements can be summarized as follows:

1. The maximum first field integral at the end of the undulator in both $x$ and $y$ must be below $4.0 \mu \mathrm{Tm}$.

2. The maximum second field integral anywhere along the undulator in both $x$ and $y$ must be below $20 \mu \mathrm{Tm}^{2}$.

3. The maximum phase error must be below $15 \mathrm{deg}$.

The first two requirements are more easily interpreted in terms of trajectory errors of a $135 \mathrm{MeV}$ beam:

1. The maximum angle of the beam at the undulator exit must be less than $8.9 \mu \mathrm{rad}$.

2. The average beam trajectory must remain straight to $44 \mu \mathrm{m}$ through the undulator.

There are other magnetic requirements in addition to these. For example, the maximum sextupole field must be below $10 \%$ of the main field at $10 \mathrm{~mm}$ from the undulator center. These requirements influence the undulator design. They will be checked in the magnetic measurements, but corrections are not expected to be necessary. Correction methods to meet these additional specifications are not discussed in this note.

\footnotetext{
${ }^{1}$ Work supported in part by the DOE Contract DE-AC02-76SF00515. This work was performed in support of the LCLS project at SLAC.

${ }^{2}$ P. Emma et al., "Laser-Heater Undulator Magnet Physics Requirements", LCLS Physics Requirements Document \#1.2-007.
} 


\section{Undulator Model}

\subsection{Field Calculations}

Rare earth permanent magnet materials have an approximately linear $B-H$ curve in the second quadrant ${ }^{3}$ and can be represented by

$$
\mathbf{B}=\mu_{0} \mathbf{H}+\mathbf{B}_{r}
$$

In this and the following expressions we use the bold font to indicate a vector. We assume $\mu \approx \mu_{0}$ for both the parallel and perpendicular block magnetization directions. Outside the block, $\mathbf{B}_{r}=0$, so equation 1 can be used at all locations with our approximations.

The fields obey the equations

$$
\begin{gathered}
\nabla \times \mathbf{H}=0 \\
\nabla \cdot \mathbf{B}=0
\end{gathered}
$$

Inserting equation 1 into equation 3 , we find

$$
\nabla \cdot \mathbf{H}=-\frac{1}{\mu_{0}} \nabla \cdot \mathbf{B}_{r}
$$

We define the magnetic volume charge density as $\rho_{m} \equiv-\frac{1}{\mu_{0}} \nabla \cdot \mathbf{B}_{r}$. Equations 2 and 4 have the same form as the equations of electrostatics.

$$
\begin{aligned}
\nabla \times \mathbf{H} & =0 \\
\nabla \cdot \mathbf{H} & =\rho_{m}
\end{aligned}
$$

The solution for $\mathbf{H}$ is derived in an analogous manner to electrostatics by using the magnetic scalar potential $\phi_{m}$, where $\mathbf{H}=-\nabla \phi_{m}$.

$$
\nabla^{2} \phi_{m}=-\rho_{m}
$$

Define a Green's function by

$$
\nabla^{2} G\left(\mathbf{x}, \mathbf{x}^{\prime}\right)=\delta\left(\mathbf{x}-\mathbf{x}^{\prime}\right)
$$

Using standard techniques, one solves for $\phi_{m}$

$$
\begin{aligned}
\int\left(G \nabla^{2} \phi_{m}-\phi_{m} \nabla^{2} G\right) d V & =\int\left(-\rho_{m} G-\phi_{m} \delta\right) d V \\
0 & =\int\left(-\rho_{m} G-\phi_{m} \delta\right) d V \\
\phi_{m}\left(x^{\prime}\right) & =\int-\rho_{m} G d V
\end{aligned}
$$

where $d V$ is a volume element and the integral is over all space. The Green's function is

$$
G\left(\mathbf{x}, \mathbf{x}^{\prime}\right)=-\frac{1}{4 \pi} \frac{1}{\left|\mathbf{x}-\mathbf{x}^{\prime}\right|}
$$

The solution for $\phi_{m}$ is

$$
\phi_{m}(\mathbf{x})=\frac{1}{4 \pi} \int \frac{\rho_{m}\left(\mathbf{x}^{\prime}\right)}{\left|\mathbf{x}^{\prime}-\mathbf{x}\right|} d V^{\prime}
$$

\footnotetext{
${ }^{3}$ K. Halbach, "Physical And Optical Properties Of Rare Earth Cobalt Materials", Nucl. Instr. and Meth. 187 (1981) 109.
} 
In this expression, care must be used at the boundaries of a permanent magnet block where $\rho_{m}$ is singular. We introduce a surface charge density to deal with the boundaries. The magnetic surface charge density is derived from the magnetic volume charge density using the definition of divergence:

$$
\nabla \cdot \mathbf{B}_{r}=\lim _{V \rightarrow 0} \frac{\oint \mathbf{B}_{r} \cdot d \mathbf{A}}{V}
$$

where the integral is over the surface of the volume and $d \mathbf{A}$ is an area element oriented toward the outer normal to the surface. Consider a small volume at the surface of a block. The magnetic charge in the volume is $\rho_{m} d V$. The definition of divergence lets us rewrite the charge in terms of a surface charge density as follows.

$$
\begin{aligned}
\rho_{m} d V & =-\frac{1}{\mu_{0}} \nabla \cdot \mathbf{B}_{r} d V \\
& =-\frac{1}{\mu_{0}} \lim _{V \rightarrow 0} \oint \mathbf{B}_{r} \cdot d \mathbf{A} \\
& =\frac{1}{\mu_{0}} \mathbf{B}_{r} \cdot d \mathbf{A} \\
& =\sigma_{m} d A
\end{aligned}
$$

i.e. $\sigma_{m}=\frac{1}{\mu_{0}} \mathbf{B}_{r} \cdot \widehat{n}$, where $\widehat{n}$ is the unit vector normal to the block, pointing out of the block.

Using the volume charge density inside the blocks and the surface charge density on the boundaries, the solution for $\phi_{m}$ is

$$
\phi_{m}(\mathbf{x})=\frac{1}{4 \pi} \int \frac{\rho_{m}\left(\mathbf{x}^{\prime}\right)}{\left|\mathbf{x}^{\prime}-\mathbf{x}\right|} d V^{\prime}+\frac{1}{4 \pi} \int \frac{\sigma_{m}\left(\mathbf{x}^{\prime}\right)}{\left|\mathbf{x}^{\prime}-\mathbf{x}\right|} d A^{\prime}
$$

The field $\mathbf{H}=-\nabla \phi_{m}$ has the following form.

$$
\mathbf{H}(\mathbf{x})=-\frac{1}{4 \pi} \int \rho_{m}\left(\mathbf{x}^{\prime}\right) \frac{\mathbf{x}^{\prime}-\mathbf{x}}{\left|\mathbf{x}^{\prime}-\mathbf{x}\right|^{3}} d V^{\prime}-\frac{1}{4 \pi} \int \sigma_{m}\left(\mathbf{x}^{\prime}\right) \frac{\mathbf{x}^{\prime}-\mathbf{x}}{\left|\mathbf{x}^{\prime}-\mathbf{x}\right|^{3}} d A^{\prime}
$$

or

$$
\mathbf{H}(\mathbf{x})=\frac{1}{4 \pi} \int \rho_{m}\left(\mathbf{x}^{\prime}\right) \frac{\mathbf{x}-\mathbf{x}^{\prime}}{\left|\mathbf{x}-\mathbf{x}^{\prime}\right|^{3}} d V^{\prime}+\frac{1}{4 \pi} \int \sigma_{m}\left(\mathbf{x}^{\prime}\right) \frac{\mathbf{x}-\mathbf{x}^{\prime}}{\left|\mathbf{x}-\mathbf{x}^{\prime}\right|^{3}} d A^{\prime}
$$

If we assume the blocks have uniform magnetization, then $\rho_{m}=0$ inside the blocks. The field is now

$$
\mathbf{H}(\mathbf{x})=\frac{1}{4 \pi} \int \sigma_{m}\left(\mathbf{x}^{\prime}\right) \frac{\mathbf{x}-\mathbf{x}^{\prime}}{\left|\mathbf{x}-\mathbf{x}^{\prime}\right|^{3}} d A^{\prime}
$$

or

$$
\begin{aligned}
\mathbf{H}(\mathbf{x}) & =\frac{1}{4 \pi} \int \frac{1}{\mu_{0}}\left[\mathbf{B}_{r}\left(\mathbf{x}^{\prime}\right) \cdot \widehat{n}\left(\mathbf{x}^{\prime}\right)\right] \frac{\mathbf{x}-\mathbf{x}^{\prime}}{\left|\mathbf{x}-\mathbf{x}^{\prime}\right|^{3}} d A^{\prime} \\
\mathbf{B}(\mathbf{x}) & =\frac{1}{4 \pi} \int\left[\mathbf{B}_{r}\left(\mathbf{x}^{\prime}\right) \cdot \widehat{n}\left(\mathbf{x}^{\prime}\right)\right] \frac{\mathbf{x}-\mathbf{x}^{\prime}}{\left|\mathbf{x}-\mathbf{x}^{\prime}\right|^{3}} d A^{\prime}
\end{aligned}
$$

These are the formulas which will be used to calculate the fields from the PPM undulator blocks. 


\subsection{Single Block Calculations}

The field $\mathbf{B}(\mathbf{x})$ is calculated numerically by dividing the surface of the magnet block into area elements. The manufacturer specifies $\mathbf{B}_{r}$ for the block. $\mathbf{B}_{r} \cdot \widehat{n}$ is calculated at each area element and multiplied by the area of the element to get the equivalent magnetic charge.

$$
q_{i}=\mathbf{B}_{r} \cdot \widehat{n} d A_{i}
$$

The charges are multiplied by the spatial factor in equation 24 and summed to calculate the field.

$$
\mathbf{B}(\mathbf{x})=\frac{1}{4 \pi} \sum_{i} q_{i} \frac{\mathbf{x}-\mathbf{x}_{i}}{\left|\mathbf{x}-\mathbf{x}_{i}\right|^{3}}
$$

The technique is illustrated in figure 1 for a single block. The charges used in the calculation are shown. The block dimensions are $0.1 \mathrm{~m}(\mathrm{x})$ by $0.1 \mathrm{~m}(\mathrm{y})$ by $0.05 \mathrm{~m}(\mathrm{z})$. The block is magnetized with $B_{r}=1.2 \mathrm{~T}$ in the $z$ direction. The $\mathbf{B}$ field is indicated with arrows. The field inside the block is also shown.

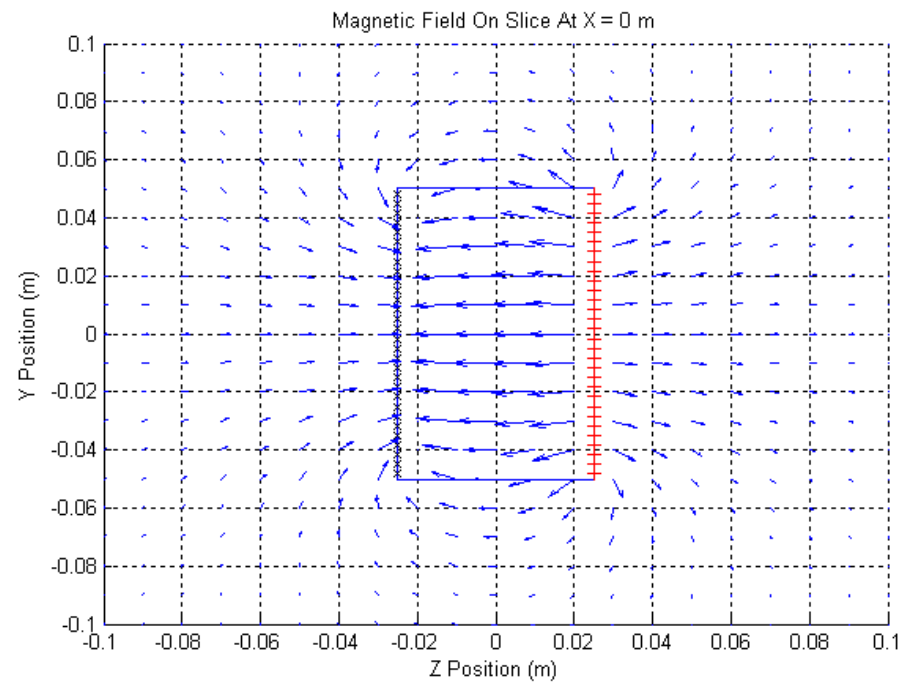

Figure 1: A permanent magnet block is modelled by a discrete set of magnetic charges. The fields from the charges are calculated and summed to get the total field at each position.

\subsection{Laser Heater Undulator Calculations}

The fields in the laser heater undulator are calculated by summing the fields from each permanent magnet block using the prescription given above. The block properties and locations are specified in an Engineering Specifications Document. ${ }^{4}$ The blocks, magnetic charges, and field vector on the axis are shown in figure 2. The $x$ and $y$ dimensions of each block were divided into 20 divisions for the charge calculation. The $z$ dimension was divided into 5 divisions. The undulator gap is 0.034 $\mathrm{m}$. The period is $0.054 \mathrm{~m}$. The remnant field is $1.2 \mathrm{~T}$. The vertical field $B_{y}$ along the line at $x=0, y=0$ is shown in figure 3 . The absolute value of the peak fields is shown in figure 4 .

\footnotetext{
${ }^{4}$ R. Carr et al., "LCLS Injector Laser Heater Undulator", LCLS Engineering Specification Document 1.2-124.
} 


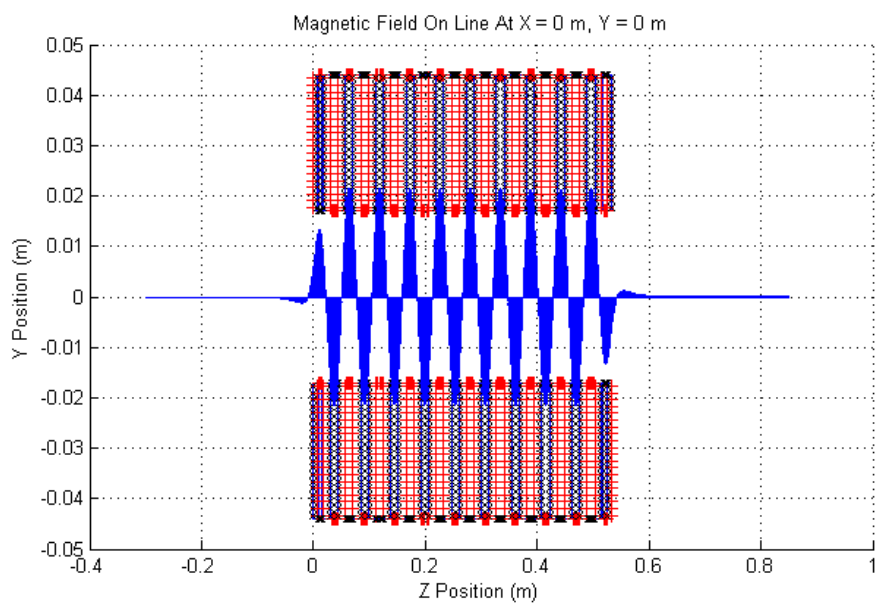

Figure 2: The laser heater undulator blocks and the magnetic charges used in the model are shown. The calculated magnetic field vector on the axis is also shown.

The beam trajectories and phases are calculated using the magnetic fields and the beam energy. ${ }^{5}$ We use a beam energy of $135 \mathrm{MeV}$ for all analysis calculations in this note. The horizontal trajectory is shown in figure 5. The heavy line is the horizontal trajectory averaged over one period. The $\mathrm{x}$-trajectory slope in the undulator is most likely a consequence of the assumptions used in the model, in particular the assumption that for the blocks $\mu=\mu_{0}$. The undulator was designed using more sophisticated models and the design trajectory does not have the slope. The trajectory slope, however, does not affect any of the results in this note. We will be concerned only with changes due to errors we introduce and shims we add to correct the errors.

The vertical trajectory is constant at $y=0$ since no horizontal fields are present on the undulator axis in the model so far. Horizontal field errors will be introduced later.

The phase errors are small for the undulator without field errors. They are shown in figure 6 . The phase errors relative to a radiation wave of $765 \mathrm{~nm}$ are shown in figure 7 . Note that the gap of the undulator was adjusted through trial and error to achieve this resonant condition.

\section{Undulator Tuning}

Having the model of the laser heater undulator allows us to introduce errors and develop tuning techniques to correct the errors. The errors are corrected by moving the permanent magnet blocks. The general procedure is to introduce an error into the model, observe the effect on the trajectories, phase, etc., find the sensitivity of the effect to block motion, and then determine the block motions required to bring the trajectories, phases, etc. within specified tolerances.

The permanent magnet blocks have tolerances on $B_{r}$ and the magnetization direction. In this section, we first determine what shim sizes are required in the undulator design to correct these errors by moving the blocks. Afterward, we discuss methods to correct trajectory errors and phase errors. We discuss the shims needed to bring the field integrals within specification. We discuss the effects of nearby dipole magnets and the effect of the Earth's field.

\footnotetext{
${ }^{5}$ Z. Wolf, "Introduction To LCLS Undulator Tuning", LCLS-TN-04-7, June, 2004.
} 


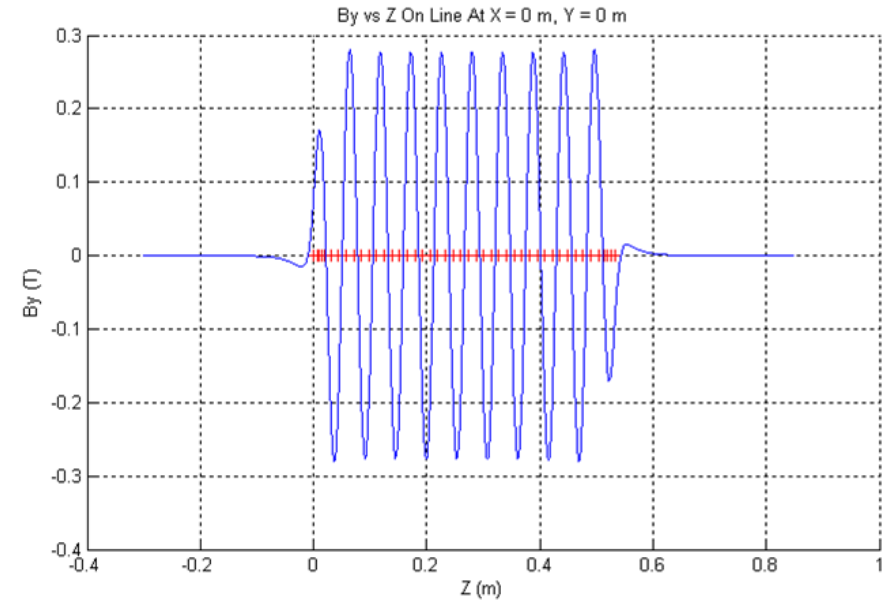

Figure 3: Magnetic field $B_{y}$ along the axis of the undulator.

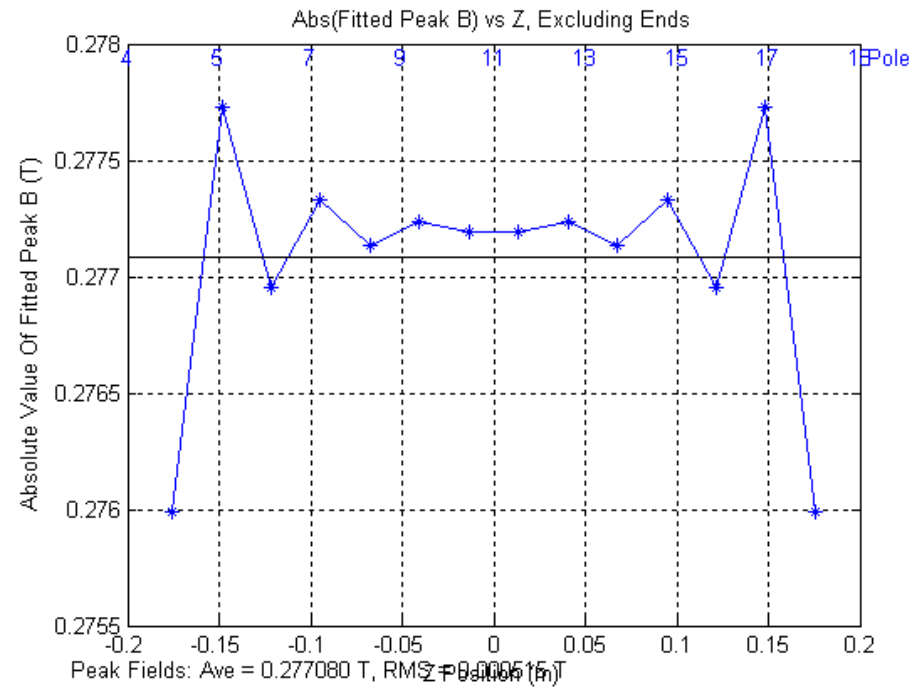

Figure 4: Absolute value of the peak fields. 


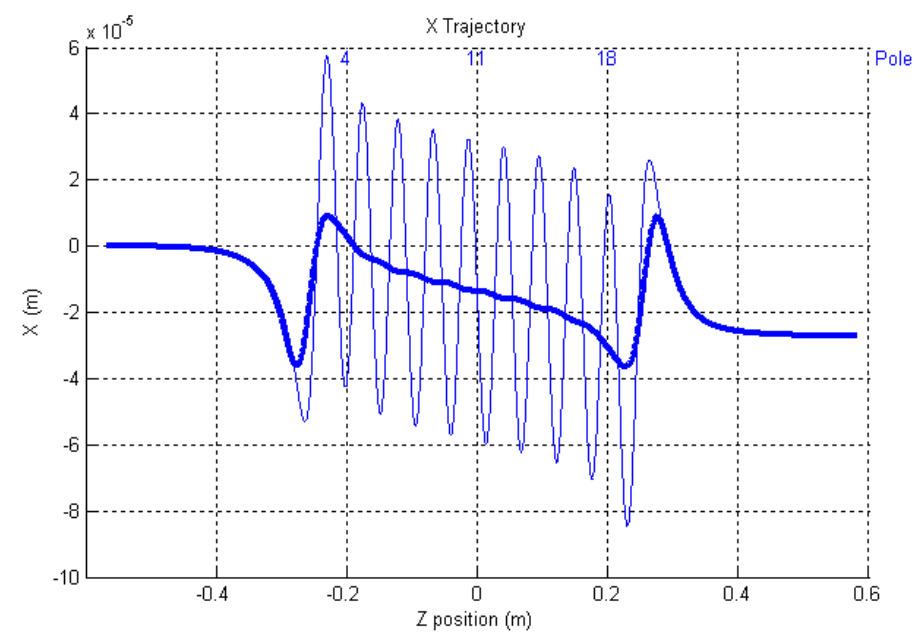

Figure 5: Horizontal trajectory in the modelled field for a beam energy of $135 \mathrm{MeV}$.

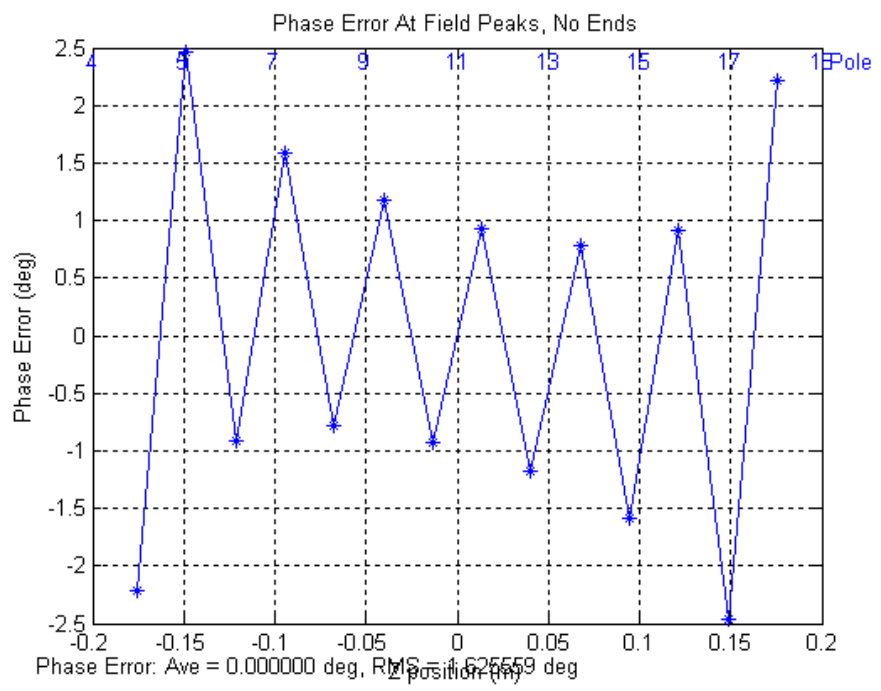

Figure 6: Phase errors in the ideal undulator. 


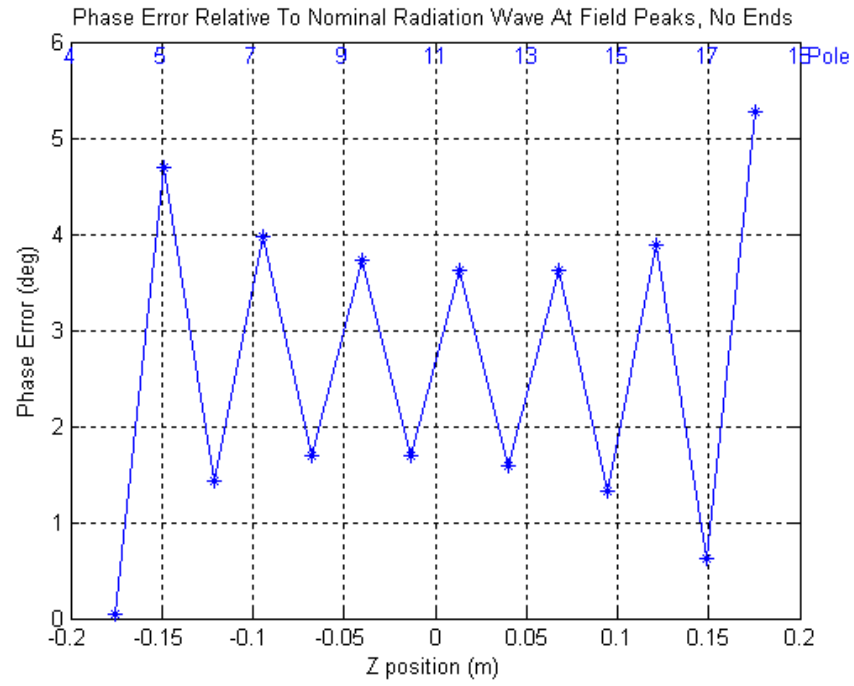

Figure 7: Phase errors relative to a radiation wave with wavelength $765 \mathrm{~nm}$. 


\subsection{Required Block Motion Range}

\subsubsection{Magnetization Magnitude Correction}

The magnet blocks are specified to have a $1 \%$ variation tolerance on their remnant field. Using the undulator model, we now determine how far a block must be moved to correct a $1 \%$ error in $B_{r}$.

Figure 8 shows the field peaks when the 20'th block (out of 41) into the undulator on the top, a vertically magnetized block, has its remnant field $1 \%$ too high. The corresponding trajectory

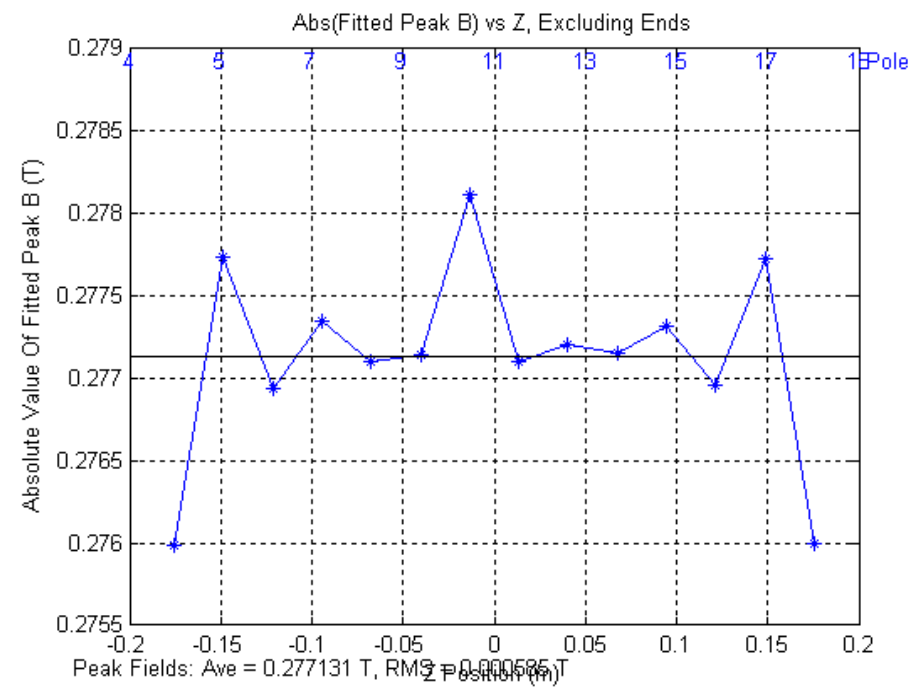

Figure 8: The 20'th block into the undulator on the top has $B_{r} 1 \%$ too large.

is shown in figure 9. The trajectory has an exit angle of $-52 \mu \mathrm{rad}$, instead of $0.49 \mu \mathrm{rad}$ for the no-error case.

To compensate for the increased $B_{r}$, the block is moved out, away from the axis. The correct amount to move the block is determined by a sensitivity analysis. For now, however, we only wish to know the range of block motions required. Figure 10 shows the trajectory when the block is moved out by $300 \mu \mathrm{m}$. The exit angle is $-1.4 \mu \mathrm{rad}$. The error is compensated. We conclude that a minimum of $300 \mu \mathrm{m}$ of transverse adjustment must be provided for each block in order to compensate for the $1 \%$ tolerance on $B_{r}$. This must be built into the undulator design for tuning.

A horizontally magnetized block was also tested. The strength of the 21'st block on the top had its $B_{r}$ increased by $1 \%$. No significant change to the trajectory was observed. No significant phase errors were introduced either. We conclude that the horizontally magnetized blocks will not require movement to compensate for their $B_{r}$ tolerance.

\subsubsection{Magnetization Direction Correction}

The magnet blocks are specified to have a 1 degree angle variation tolerance on their magnetization direction. Magnetization direction errors cause transverse fields which affect the beam. In figure 11 , the vertically magnetized 20 'th block into the undulator on the top has a 1 degree magnetization rotation counterclockwise about the beam direction. The resulting vertical trajectory has a resulting large excursion as shown in figure 12.

In order to correct the magnetization direction error, the block is rotated so the main field obtains a transverse component. It was determined empirically that the block must be rotated 


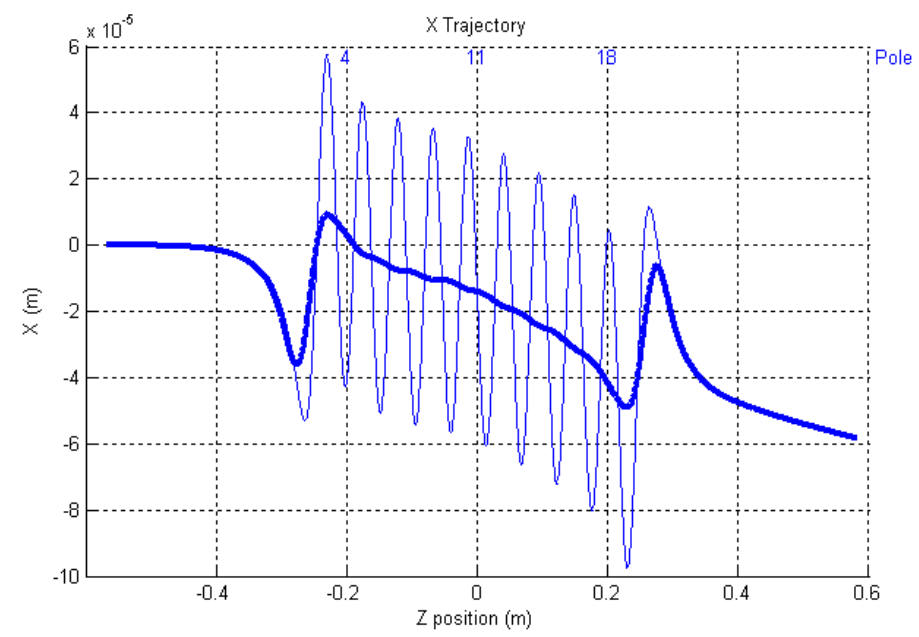

Figure 9: Horizontal trajectory with a $1 \%$ remnant field error in the 20 'th block into the undulator on the top.

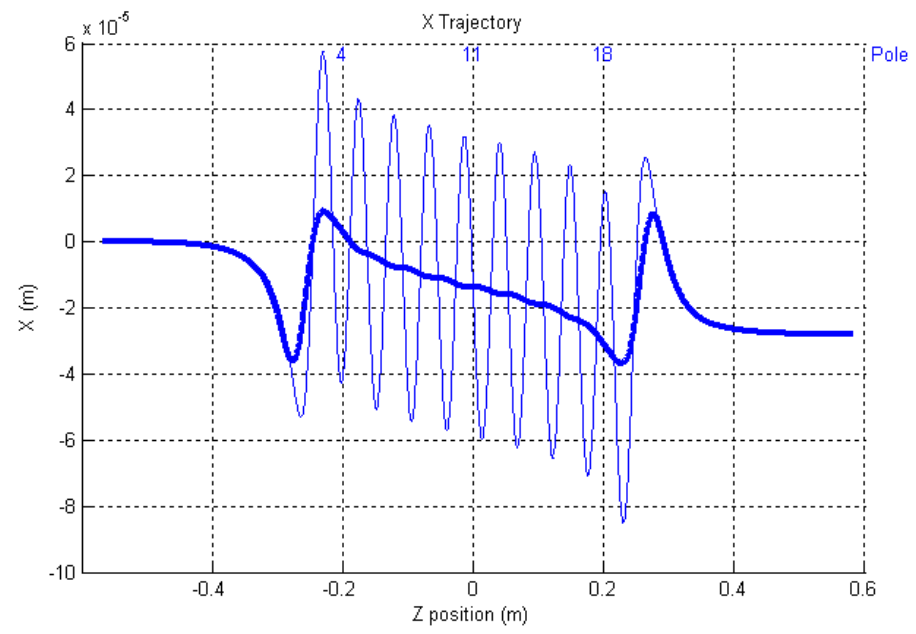

Figure 10: The 20'th block into the undulator on the top with $B_{r} 1 \%$ too large has been moved out by 300 microns. The resulting horizontal trajectory is shown. 


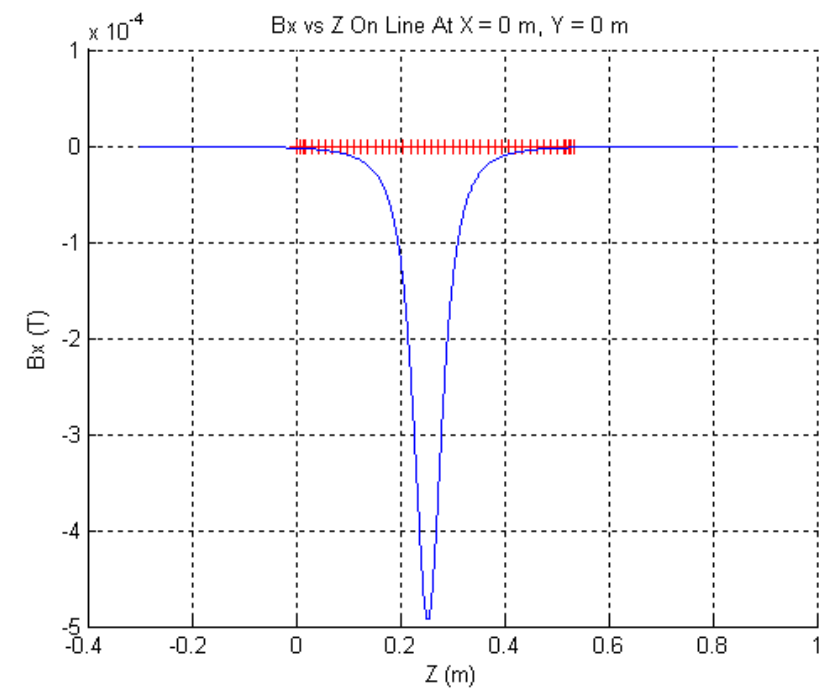

Figure 11: The 20'th block into the undulator on the top has a 1 degree magnetization rotation counterclockwise about the beam direction. This figure shows the resulting transverse field $B_{x}$ as a function of position $z$ along the undulator.

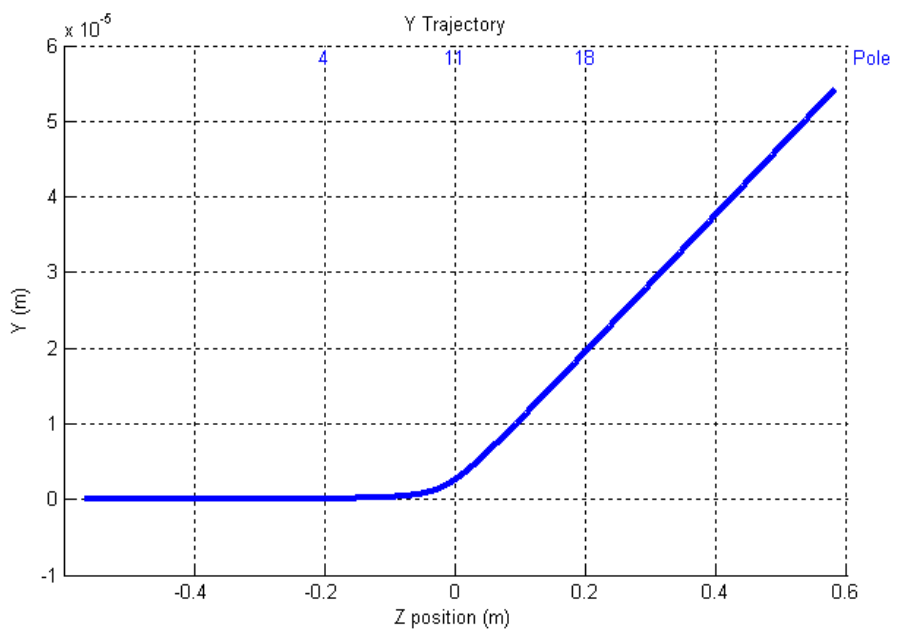

Figure 12: This figure shows the vertical trajectory with the 1 degree magnetization direction error in the 20 'th block on the top. 


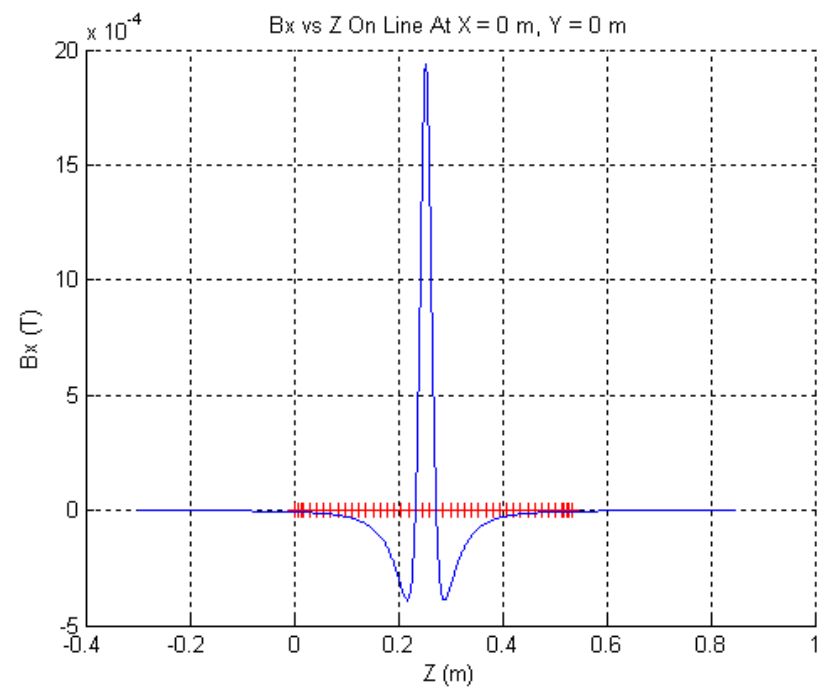

Figure 13: The 20'th block on the top with the magnetization direction error was rotated 2 degrees counterclockwise about the beam direction. This figure shows the resulting transverse field. 
approximately 2 degrees counterclockwise about the beam direction to correct the error. Figure 13 shows the resulting transverse field. The resulting vertical trajectory is shown in figure 14 . The plot was made with the same vertical scale as figure 12 for comparison. The 2 degree rotation made the trajectory an order of magnitude smaller and approximately corrected the error. The undulator design must provide for at least 2 degree block rotations.

To explain the longitudinal behavior of the transverse fields, note that a magnetization direction error places charges on the sides of the block, far from the beam. Rotating the block, however, primarily has the effect of rotating the plane of charges on the block face near the beam. The longitudinal extent of these two effects is different.

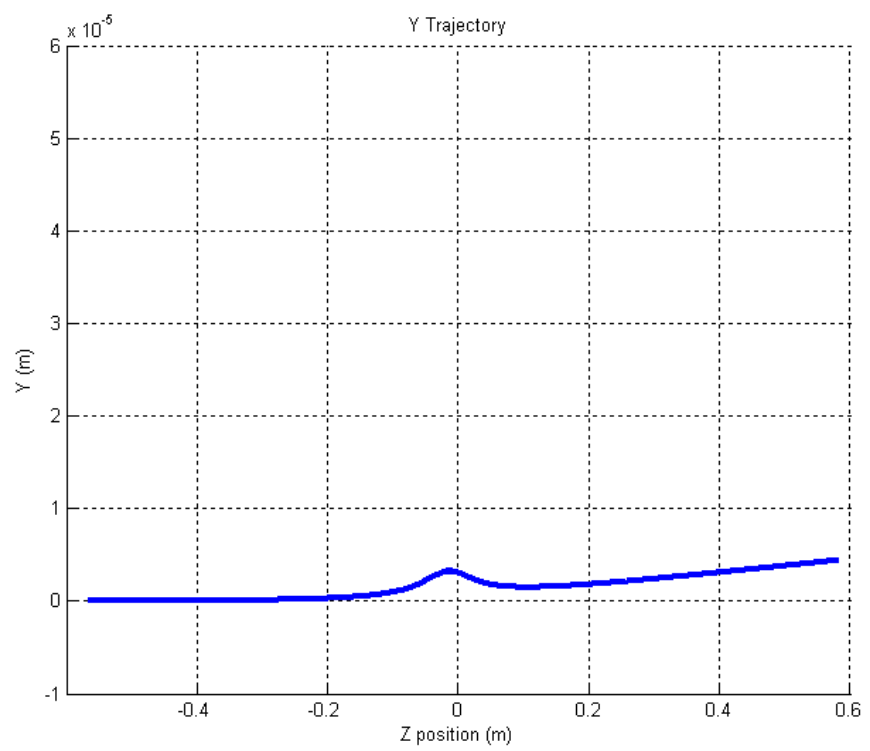

Figure 14: Vertical trajectory after the block with the 1 degree magnetization direction error was rotated by 2 degrees.

When rotating the blocks, it is important to perform the rotation such that the center of the block face near the beam is stationary. This is illustrated in figure 15. The blocks are shimmed on their back faces. Adjusting the shims to solely rotate the block translates the face of the block near the undulator gap horizontally and vertically. This causes additional horizontal and vertical field errors. The blocks must be shimmed such that the center of the face near the undulator gap does not move.

We wish to know the approximate shim size required to correct the 1 degree magnetization direction error. We determined that the block must be rotated approximately 2 degrees. The block is $0.06 \mathrm{~m}$ in width. To do the rotation, one shim must be increased in thickness by approximately $0.001 \mathrm{~m}$ and the other shim decreased in thickness by approximately $0.001 \mathrm{~m}$. This range of shim sizes must be built into the design of the undulator. The required horizontal translation and horizontal alignment of the face near the undulator gap must also be built into the design of the undulator.

Magnetization direction errors in horizontally magnetized blocks were also studied. When a horizontally magnetized block has a 1 degree magnetization direction error giving a horizontal $\left(\mathbf{B}_{r}\right)_{x}$ component, magnetic charges are introduced on the outer sides of the block giving the same horizontal field distribution and trajectory as shown above for the vertically magnetized block error 

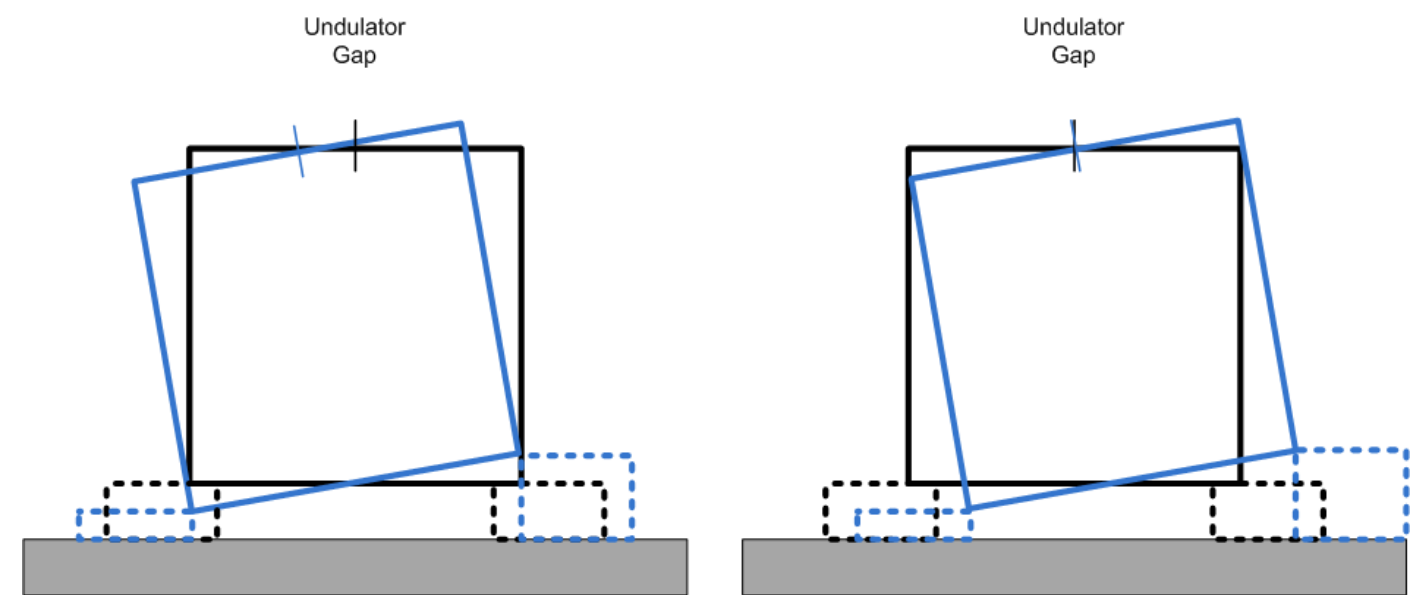

Figure 15: The shims used to adjust the block position are on the back side of the block. Rotating the block by solely adjusing the shims translates the face of the block near the undulator gap. This is shown on the left. Further transverse fields are produced. The block must also be translated horizontally and vertically so the center of the face near the undulator gap does not move. This is shown on the right.

case. The error is corrected by rotating an adjacent vertically magnetized block as discussed above. When a horizontally magnetized block has a 1 degree magnetization direction error giving a vertical $\left(\mathbf{B}_{r}\right)_{y}$ component, magnetic charges are introduced on the faces of the block near the gap, as for a vertically magnetized block. From the sine of the angle, the strength of the charges is $1.7 \%$ of the charges for a vertically magnetized block. Indeed, the exit angle of the horizontal trajectory is $91 \mu \mathrm{rad}$, about a factor of 1.7 times larger than when the vertically magnetized block had a $1 \%$ magnetization error. This error is corrected by moving adjacent vertically magnetized blocks toward or away from the gap, as for the magnetization magnitude error case.

\subsection{X-Trajectory Tuning}

We now determine the x-trajectory angle change as a function of block motion. Both upper and lower blocks will be moved in order to minimize gradients on the axis and thus maximize the undulator good field region. This is illustrated in figure 16.

The 20'th blocks into the undulator, both upper and lower, were moved for this study. Figure 17 shows the peak fields when the 20'th upper and lower blocks are moved $400 \mu \mathrm{m}$ away from the gap. The resulting horizontal trajectory is shown in figure 18. The exit angle change in the trajectory is $134 \mu \mathrm{rad}$.

We wish to know the x-trajectory angle change as a function of block motion. This is shown in figure 19. The trajectory angle changes linearly with block motion distance. The x-trajectory angle changes by $0.334 \mu \mathrm{rad}$ per $\mu \mathrm{m}$ of block motion. Both upper and lower blocks are moved simultaneously. The result applies for motion both into and out of the gap. 


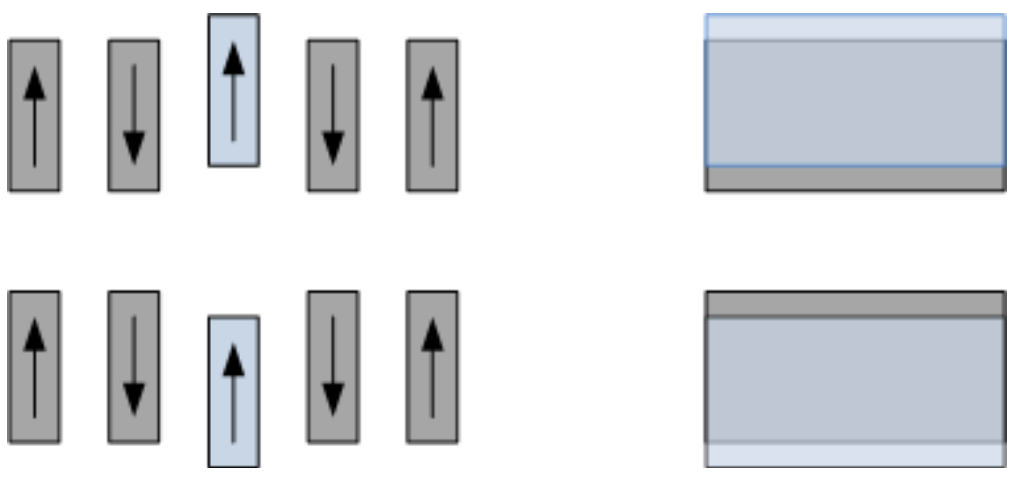

Figure 16: Moving both upper and lower vertically magnetized blocks changes the vertical magnetic field. This is used to adjust the x-trajectory.

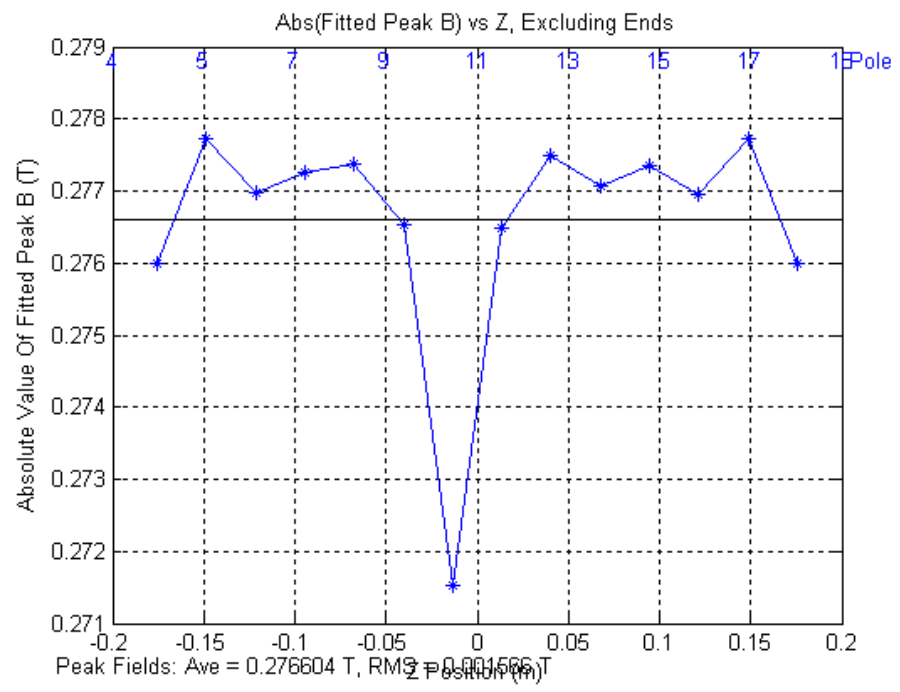

Figure 17: The 20'th blocks into the undulator, both upper and lower, were moved 400 microns away from the gap. The absolute value of the peak fields is shown. 


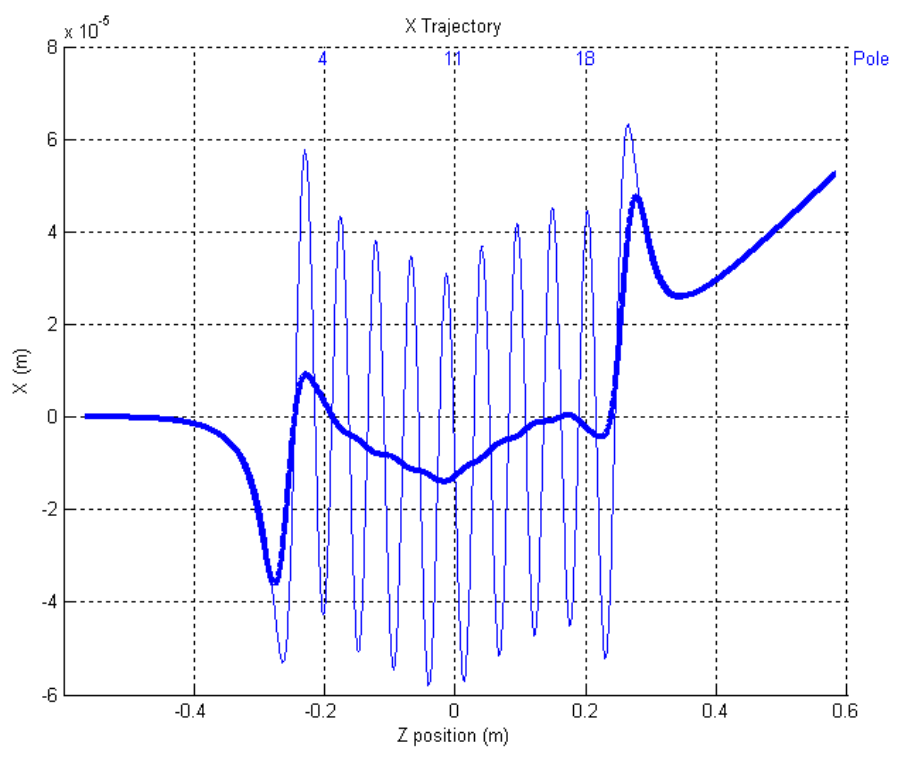

Figure 18: This figure shows the horizontal trajectory when the 20 'th blocks into the undulator are moved 400 microns away from the gap.

\section{X-Trajectory Shim Sensitivity}

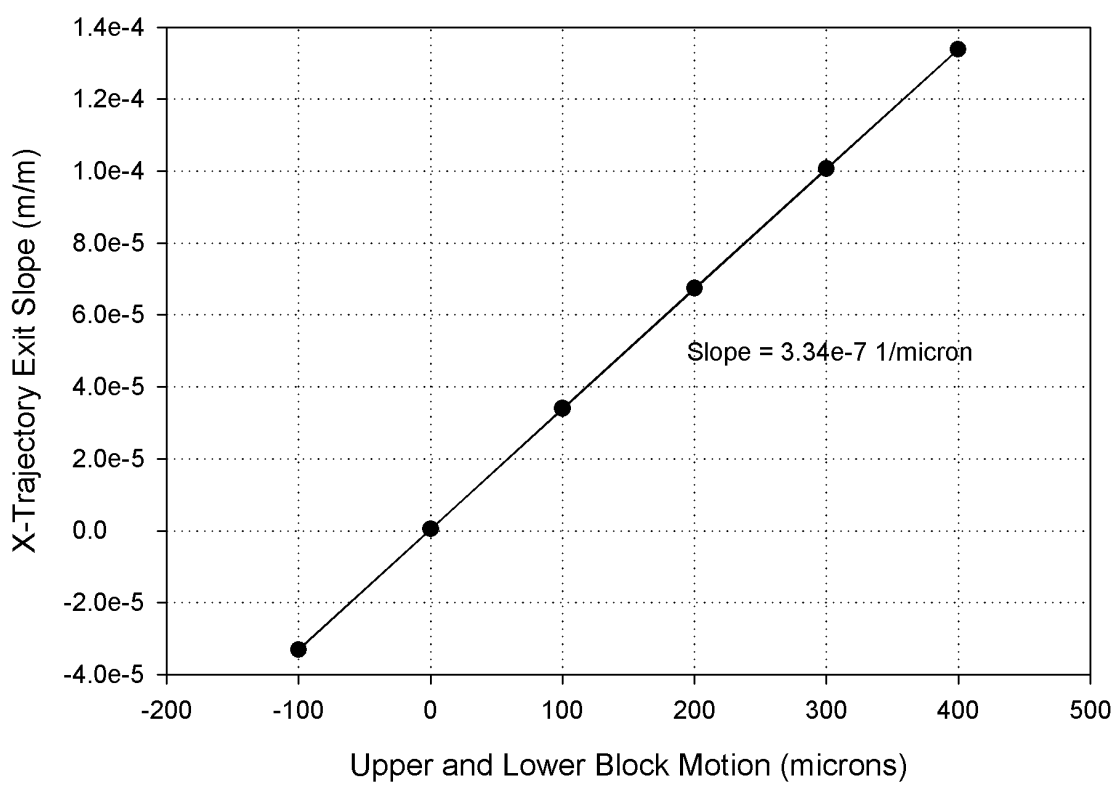

Figure 19: X-trajectory angle change as a function of distance both the upper and lower blocks are moved away from the gap. Negative motion is toward the gap. 


\subsection{Y-Trajectory Tuning}

The y-trajectory is changed by creating a horizontal magnetic field. This is done by rotating the magnet blocks. Both upper and lower blocks are rotated in order to minimize gradients and preserve a large good field region. The block motion is illustrated in figure 20.
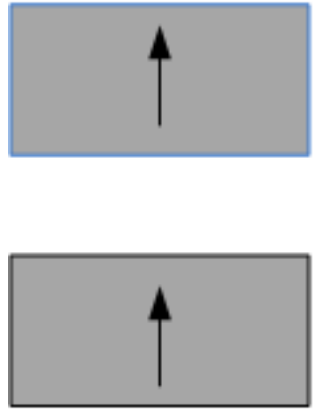
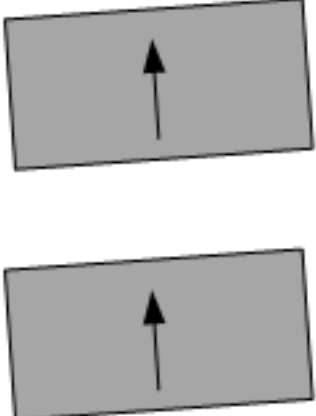

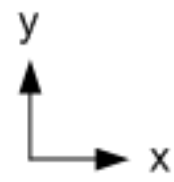

Figure 20: Upper and lower blocks are rotated to adjust the vertical trajectory.

The angle change of the y-trajectory was studied as a function of block rotation angle. Rotating the 20 'th blocks into the undulator, both upper and lower, by 0.5 degree produced the horizontal field along the undulator shown in figure 21. The resulting y-trajectory is shown in figure 22. The

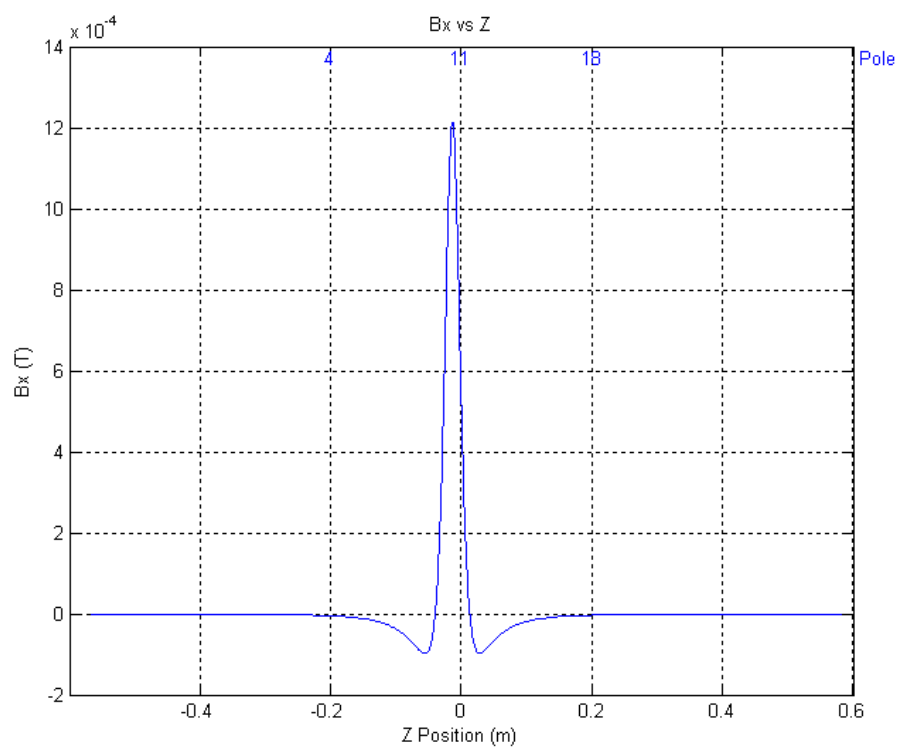

Figure 21: The 20'th blocks into the undulator, both upper and lower, were rotated by 0.5 degree. This figure shows the resulting horizontal magnetic field.

y-trajectory angle change is $-41.9 \mu \mathrm{rad}$.

The angle change in the y-trajectory for various block rotations is shown in figure 23. A beam energy of $135 \mathrm{MeV}$ was used. The upper and lower 20'th blocks into the undulator were both rotated through the same angle. The exit angle of the y-trajectory, which is the same as the trajectory 


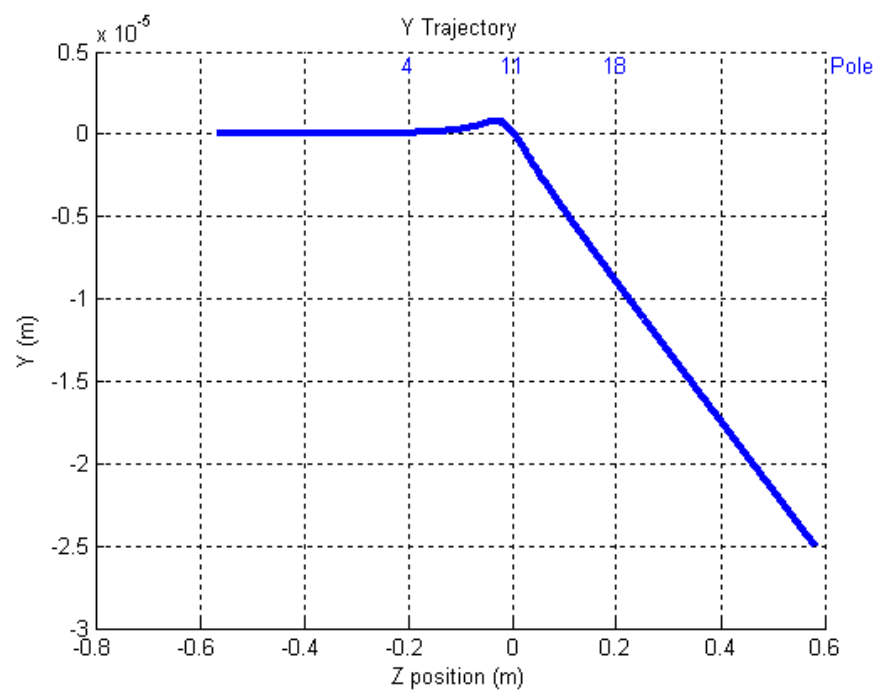

Figure 22: Vertical trajectory resulting from a 0.5 degree rotation of the 20 'th blocks into the undulator.

slope change due to the rotation, is shown in the plot. The angle change is well behaved and linear. The sensitivity of the $\mathrm{y}$-trajectory angle to block rotation is $-83.7 \mu \mathrm{rad}$ per degree of block rotation.

\subsection{Phase Tuning}

The phase is adjusted by changing the strength of neighboring vertically magnetized blocks. Four blocks are moved, two on the top, and two on the bottom of the undulator. This produces a local increase in the beam undulation if the blocks are moved toward the gap resulting in a positive phase shift. If the blocks are moved away from the gap, the fields get weaker and the undulation gets smaller. A negative phase shift results. The block motions are illustrated in figure 24. The resulting change in the peak fields is shown in figure 25 . The 20 'th and 22'nd blocks into the undulator, both upper and lower, were moved $400 \mu \mathrm{m}$ away from the gap. The resulting phase of a $135 \mathrm{MeV}$ beam relative to a radiation wave of $765 \mathrm{~nm}$ is shown in figure 26. A -9.09 degree phase shift results. The x-trajectory has a small shift, but otherwise no changes to the trajectories result. The $\mathrm{x}$-trajectory with the blocks moved is shown in figure 27 .

The phase shift as a function of block motion is shown in figure 28. Note that the phase can be increased by moving the blocks into the gap. The phase is decreased by moving the blocks away from the gap. The sensitivity is -0.0227 degree of phase change per $\mu \mathrm{m}$ of block motion away from the gap. 


\section{Y-Trajectory Shim Sensitivity}

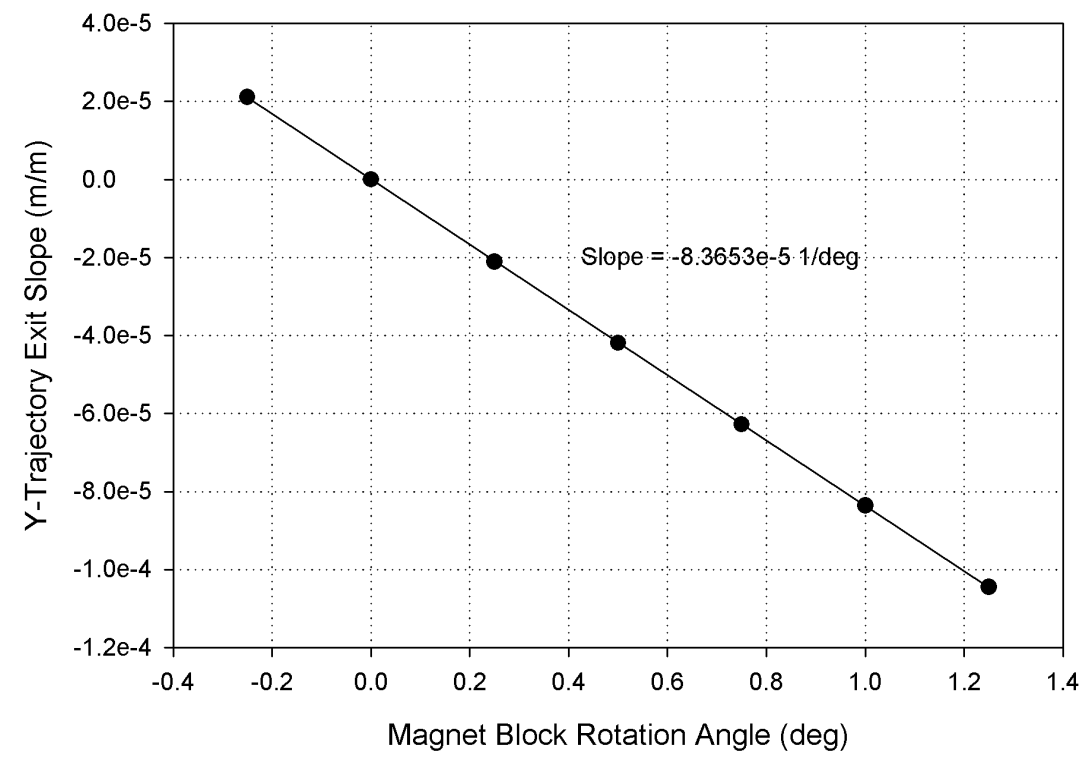

Figure 23: Y-trajectory angle change as a function of upper and lower block rotation angle.

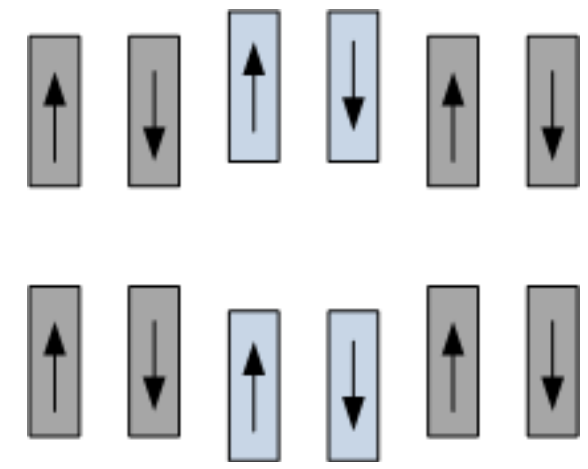

Figure 24: A phase shift is produced by moving four blocks. 


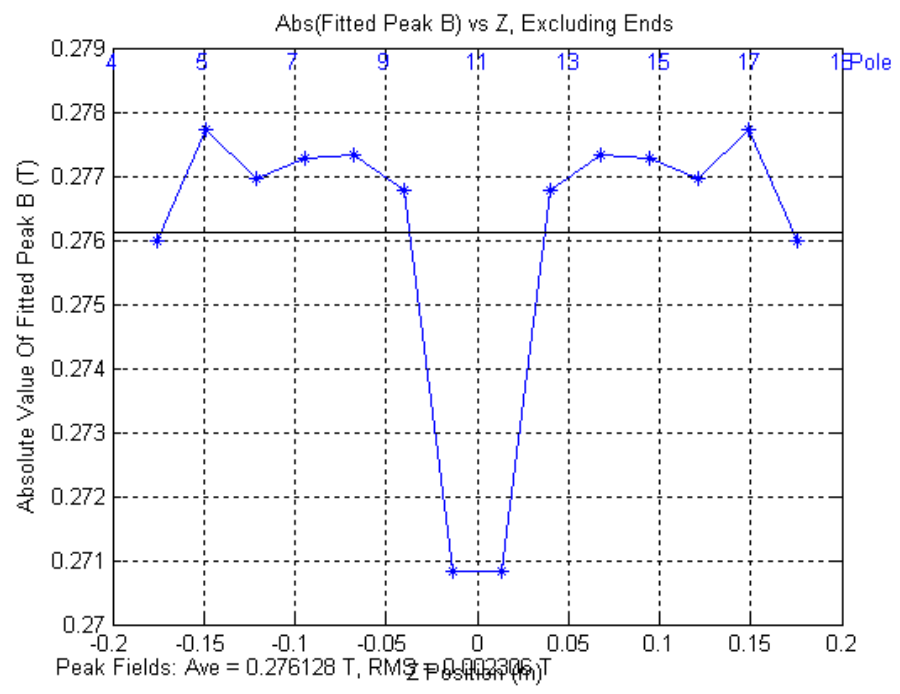

Figure 25: The peak fields of the 20'th and 22'nd blocks into the undulator, both upper and lower, were reduced when they were moved 400 microns away from the gap.

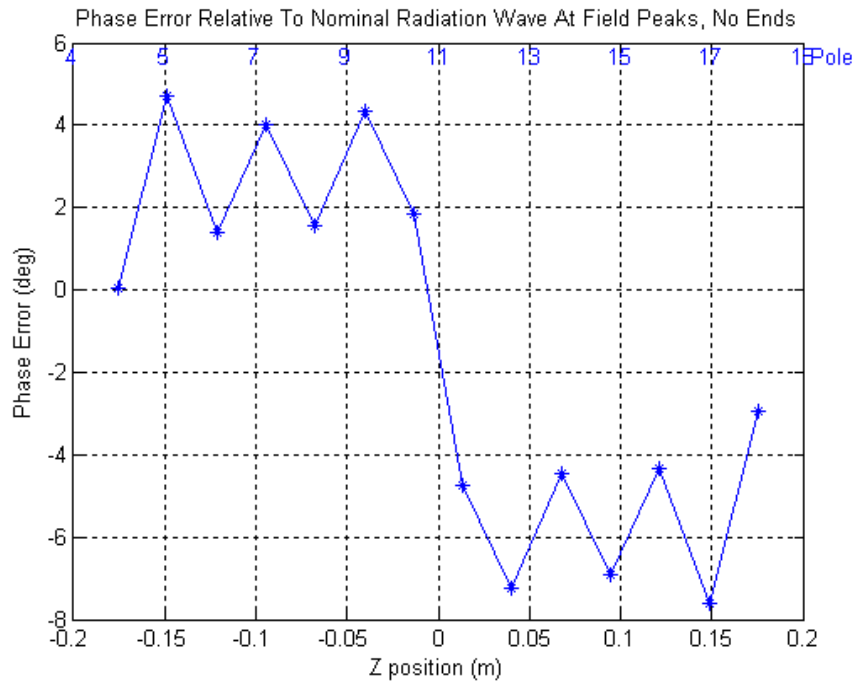

Figure 26: Phase change resulting from moving the 20'th and 22'nd blocks into the undulator, both upper and lower, by 400 microns away from the gap. 


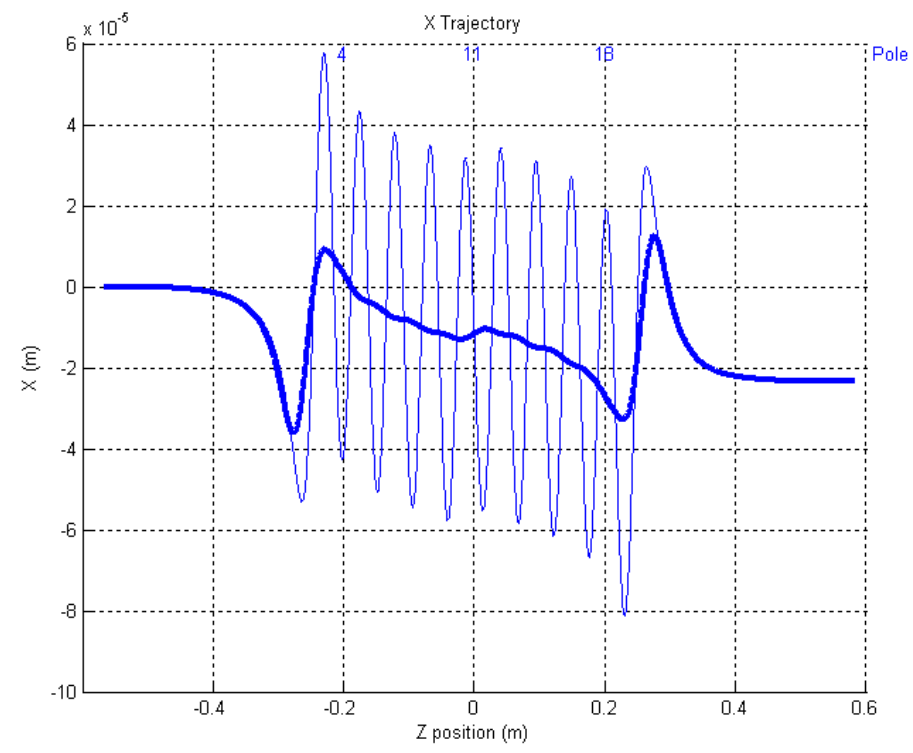

Figure 27: The $x$-trajectory has a small perturbation when the 20'th and 22'nd blocks into the undulator, both upper and lower, are moved 400 microns away from the gap for a phase adjustment.

\section{Phase Shim Sensitivity}

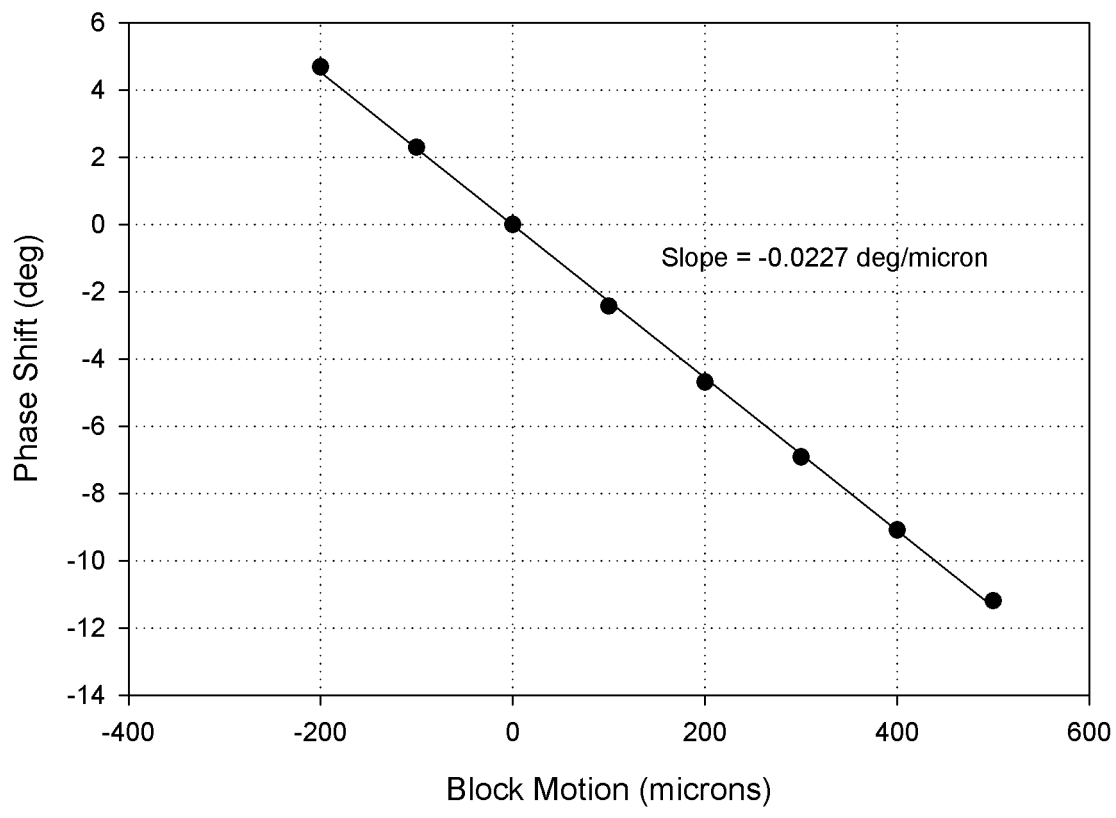

Figure 28: Phase shift as a function of block motion. Negative motion is into the gap. Positive motion is away from the gap. 


\subsection{Field Integrals}

We now wish to determine the smallest block motions which are required. Mechanical shims provide limited resolution, down to approximately $10 \mu \mathrm{m}$. The most sensitive shimming requirements come from the field integral specifications. We check that we can meet the specifications with the resolution of the shims.

\subsubsection{First Field Integrals}

As noted previously, the first field integrals of both $B_{x}$ and $B_{y}$ must be less than $4.0 \mu \mathrm{Tm}$. This translates into a trajectory exit angle below $8.9 \mu \mathrm{rad}$ for a $135 \mathrm{MeV}$ beam. In order to bring the first field integrals within specification, an angle change is made near the exit end of the undulator. This allows the first field integral to be changed without a significant change to the second field integral.

For the horizontal trajectory, upper and lower blocks are moved either in or away from the gap to change the angle. The sensitivity of trajectory angle change with block motion was found to be $0.334 \mu \mathrm{rad}$ per $\mu \mathrm{m}$ of block motion. Thus, block motions smaller than and up to $26.6 \mu \mathrm{m}$ will be required to meet the exit angle tolerance.

For the vertical trajectory, upper and lower blocks are rotated to change the angle. The sensitivity of trajectory angle change to block rotation is $83.7 \mu \mathrm{rad}$ per degree of block rotation. In order to meet the first integral requirement, block rotations smaller than and up to 0.11 degree will be required. For the $0.06 \mathrm{~m}$ wide blocks, positive and negative changes of shim thickness of $58 \mu \mathrm{m}$ are required on either side of the block.

The first field integral specifications can be met with mechanical shims. The shim resolution is close to, but smaller than, the block motion resolution required to meet the specification.

\subsubsection{Second Field Integrals}

The second field integrals of both $B_{x}$ and $B_{y}$ must be less than $20 \mu \mathrm{Tm}^{2}$. This translates into an exit position offset less than $44 \mu \mathrm{m}$ for a $135 \mathrm{MeV}$ beam. The exit position of the beam is adjusted by giving the beam a small angle change at the undulator entrance. The undulator is approximately $0.55 \mathrm{~m}$ long. The angle change required to change the exit position by $44 \mu \mathrm{m}$ is $80 \mu \mathrm{rad}$. We now determine the shim size changes required to make this angle change at the entrance. The result will give the required shim resolution to meet the second field integral tolerance.

The horizontal trajectory slope is changed by moving the blocks toward or away from the beam line. The sensitivity of the trajectory angle change is $0.334 \mu \mathrm{rad}$ per $\mu \mathrm{m}$ of block motion. With this sensitivity, block motions of $240 \mu \mathrm{m}$ are required to move the beam exit position by $44 \mu \mathrm{m}$. The shims easily provide the resolution to achieve this tolerance.

The vertical trajectory slope is changed by rotating the blocks The trajectory angle change required to change the exit position by $44 \mu \mathrm{m}$ is again $80 \mu \mathrm{rad}$. The sensitivity of trajectory angle change to block rotation is $83.7 \mu \mathrm{rad}$ per degree of block rotation. With this sensitivity, a rotation of 0.96 degree is required to move the exit beam position by $44 \mu \mathrm{m}$. For the $0.06 \mathrm{~m}$ wide blocks, positive and negative changes of shim thickness of $503 \mu \mathrm{m}$ are required on either side of the block. The shims easily provide the resolution to achieve the horizontal field integral tolerance.

\subsection{Effect Of Nearby Magnets}

A dipole will be placed on either side of the undulator in the tunnel. We wish to determine whether the effect of the nearby dipoles must be accounted for in the tuning. We model the effect of each dipole by setting the undulator field to zero for all points which would be in the dipole. Figure 29 shows the layout of the undulator and the dipoles. The figure shows dimensions in inches. The region between dipoles extends 17.24 inches on either side of the undulator center. Converting to 
meters, the undulator field for this study was set to zero for positions greater than $0.438 \mathrm{~m}$ from the undulator center. When this was done, the horizontal trajectory exit angle did not change. This is due to the antisymmetric undulator field being truncated symmetrically about the undulator center. The exit position of the horizontal trajectory moved by $12.6 \mu \mathrm{m}$ toward positive $x$. The sign agrees with the negative $B_{y}$ undulator field being truncated on the undulator entrance end. The change in angle due to the dipole is $12.6 \mu \mathrm{m} / 1.02 \mathrm{~m}=12.4 \mu \mathrm{rad}$.

Looking at the undulator fields at the location of the dipole gives the same conclusion. With no dipole in place and an undulator gap of $0.034 \mathrm{~m}$, the field $0.438 \mathrm{~m}$ from the undulator center is $-1.2 \mathrm{G}$ on the entrance end $(+1,2 \mathrm{G}$ on the exit end). The field goes to $-0.14 \mathrm{G}$ at a distance $0.567 \mathrm{~m}$ from the undulator center, but in a non-linear manner. The corresponding field integral performed using a simple numerical integration is $5.9 \mu \mathrm{Tm}$. The angle change of a $135 \mathrm{MeV}$ beam in this integrated field is $13 \mu \mathrm{rad}$, which agrees with the calculation given above.

One may wonder if the trajectory angle changes as the undulator gap is opened. The field at the dipole location is larger. Performing the simulation with an undulator gap of $0.1 \mathrm{~m}$, with and without truncating the field at the dipole location, one finds that the exit position of the beam changes by $15.5 \mu \mathrm{m}$ toward positive $x$ due to the field truncation. The change in angle due to the dipole is $15.5 \mu \mathrm{m} / 1.02 \mathrm{~m}=15.2 \mu \mathrm{rad}$. The field at the dipole is $-1.35 \mathrm{G}$. This is a small change from the case of the nominal gap. The undulator field interaction with the dipole will not significantly change the beam steering as the gap is opened.

The beam position will be monitored by OTR screens on either side of the undulator. Correctors will be used to compensate for the trajectory angle change caused by the interaction of the dipole steel with the undulator field. The angle change due to the dipole is very small. No special measurements with steel near the undulator need to be made.

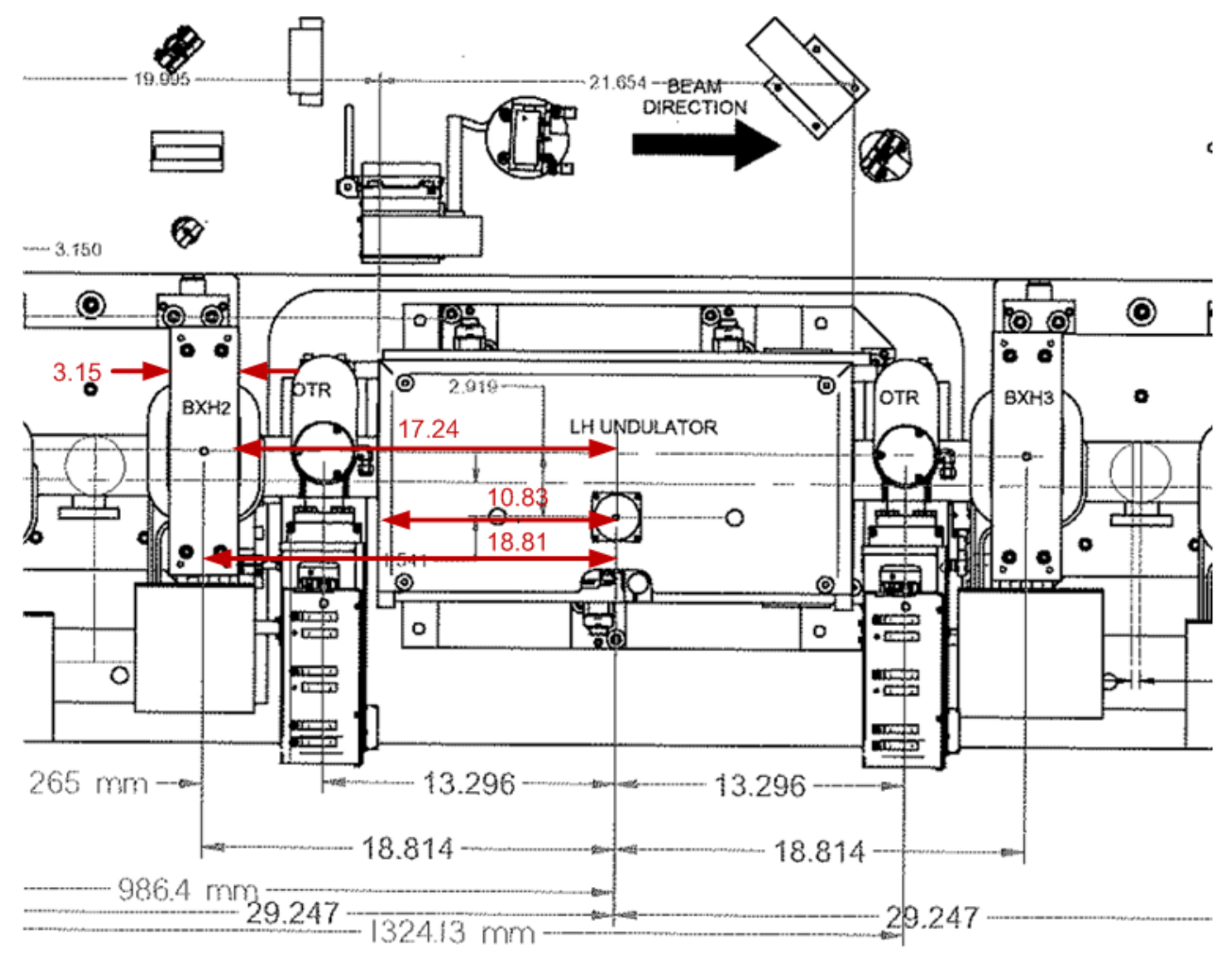

Figure 29: Dipoles will be placed near the undulator in the tunnel. 


\subsection{Effect Of The Earth's Magnetic Field}

The laser heater undulator has no permeable components to shield the Earth's magnetic field. Both the vertical and horizontal components of the Earth's field will appear in the undulator gap. The undulator will be tuned to compensate for the Earth's field at the location of the tuning. Differences in the Earth's field between the tunnel location and the tuning location, however, will cause trajectory errors. A number of measurements have been previously carried out in order to estimate the difference in the Earth's field between the tunnel and the tuning laboratory. ${ }^{6}$ The estimated difference is $0.08 \mathrm{G}$ for both the vertical and horizontal fields. A further difference is that the tuning laboratory is laid out parallel to the linac, whereas, the laser heater undulator will be placed in the rotated injector tunnel. The vertical field will not be influenced, but the horizontal field will be. No shielding or other remedy is proposed for the Earth's field difference. We merely model these field differences and estimate the size of the effect. Correctors will be required to compensate the beam trajectory.

A $0.1 \mathrm{G}$ vertical field difference causes the horizontal trajectory to go from figure 5 to figure 30 . The exit angle is $26 \mu \mathrm{rad}$, while the requirement is that it be less than $8.9 \mu \mathrm{rad}$. Correctors will be

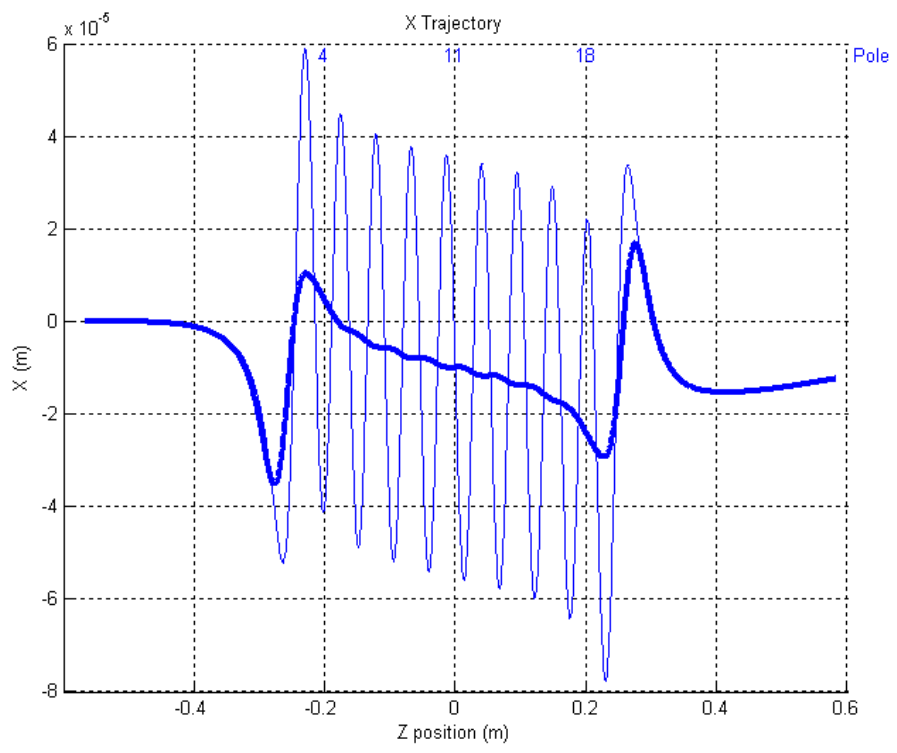

Figure 30: A $0.1 \mathrm{G}$ difference in the Earth's vertical field between the tuning laboratory and the tunnel produces the $\mathrm{x}$-trajectory shown.

required to remove the trajectory angle. Note that it is possible that the field difference between the laboratory and the tunnel will be larger.

A $0.1 \mathrm{G}$ horizontal field difference causes the vertical trajectory to go from a straight line at zero to that shown in figure 31 . The exit angle is $26 \mu \mathrm{rad}$, as for the horizontal trajectory, and is out of tolerance. A horizontal field correction will be required in the tunnel.

The maximum Earth field difference occurs when the undulator is tuned in one orientation and turned 180 degrees in use. In this case, the horizontal component of the Earth's field will be twice as large in the undulator. Measurements of the horizontal component of the Earth's field show

\footnotetext{
${ }^{6}$ K. Hacker et al., "Earth's Magnetic Field Measurements For The LCLS Undulators", LCLS-TN-05-4, February, 2005.
} 


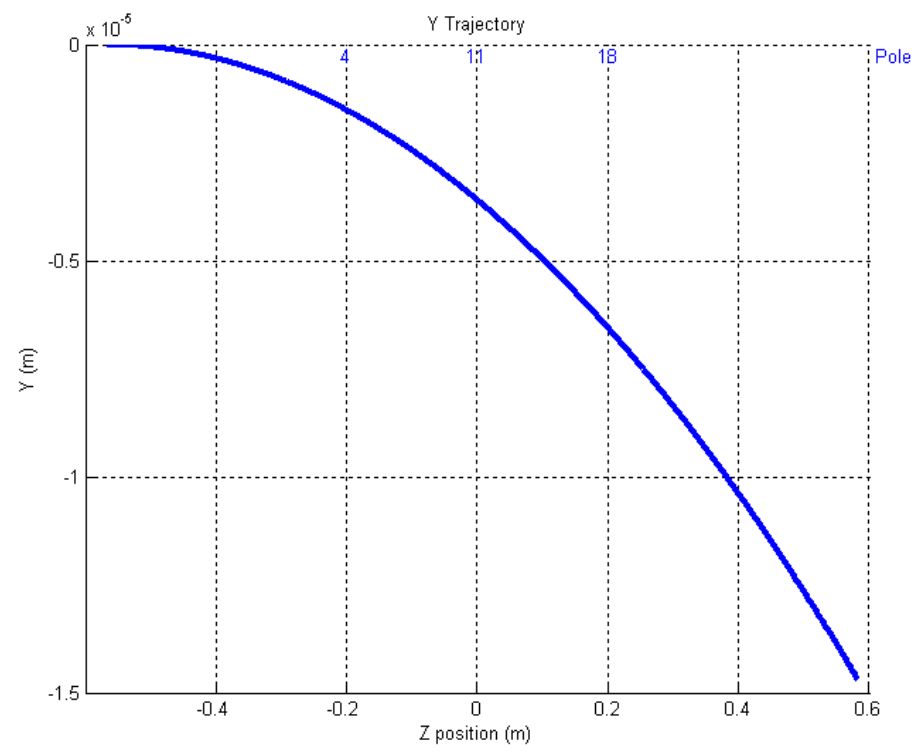

Figure 31: A horizontal field difference of $0.1 \mathrm{G}$ causes the vertical trajectory shown here.

that it is typically around $0.25 \mathrm{G} .{ }^{7}$ If the undulator is tuned to cancel this $0.25 \mathrm{G}$ and then turned around, the $0.25 \mathrm{G}$ field build into the undulator from tuning will add to the $0.25 \mathrm{G}$ field from the Earth to give a $0.5 \mathrm{G}$ horizontal field. The resulting vertical trajectory is shown in figure 32 . In this figure, the horizontal field is $0.25 \mathrm{G}$ outside the undulator and $0.5 \mathrm{G}$ inside. The $44 \mu \mathrm{m}$ trajectory straightness tolerance is exceeded. The average angle can be removed with corrector magnets. The remaining sagitta is shown in figure 33. The sagitta over the extended range of the plot is approximately $15 \mu \mathrm{m}$, which is within the $44 \mu \mathrm{m}$ trajectory straightness tolerance for the undulator. The sagitta in the $0.55 \mathrm{~m}$ long undulator is approximately $5 \mu \mathrm{m}$, which is much smaller than the straightness tolerance. We conclude that the Earth's field can be adequately compensated with external corrector magnets and that the external correctors will likely be required.

${ }^{7}$ ibid. 


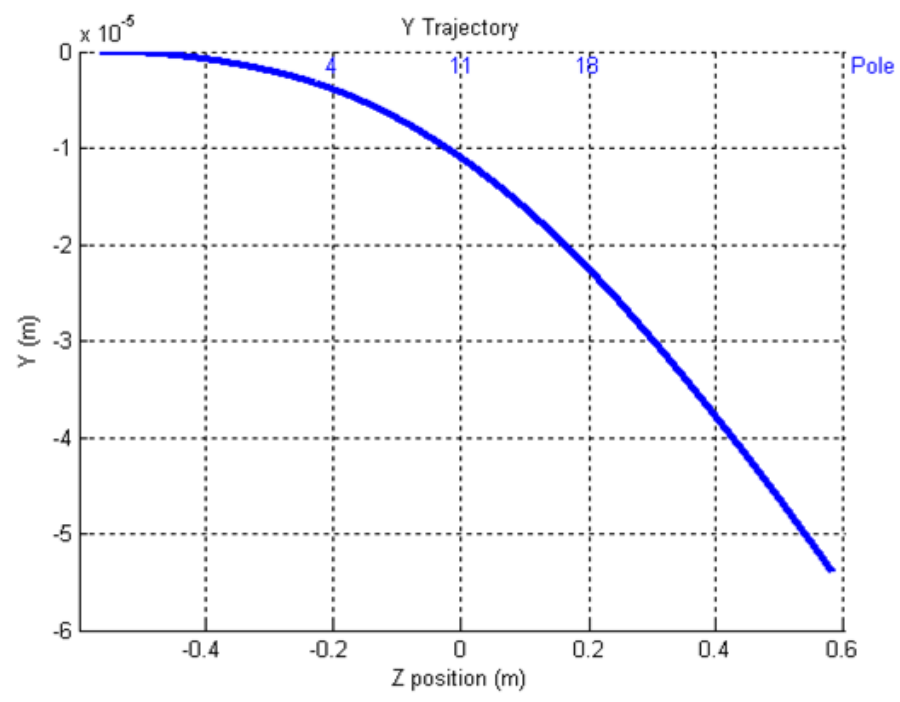

Figure 32: A horizontal field of $0.25 \mathrm{G}$ outside the undulator and $0.5 \mathrm{G}$ inside the undulator produces this vertical trajectory.

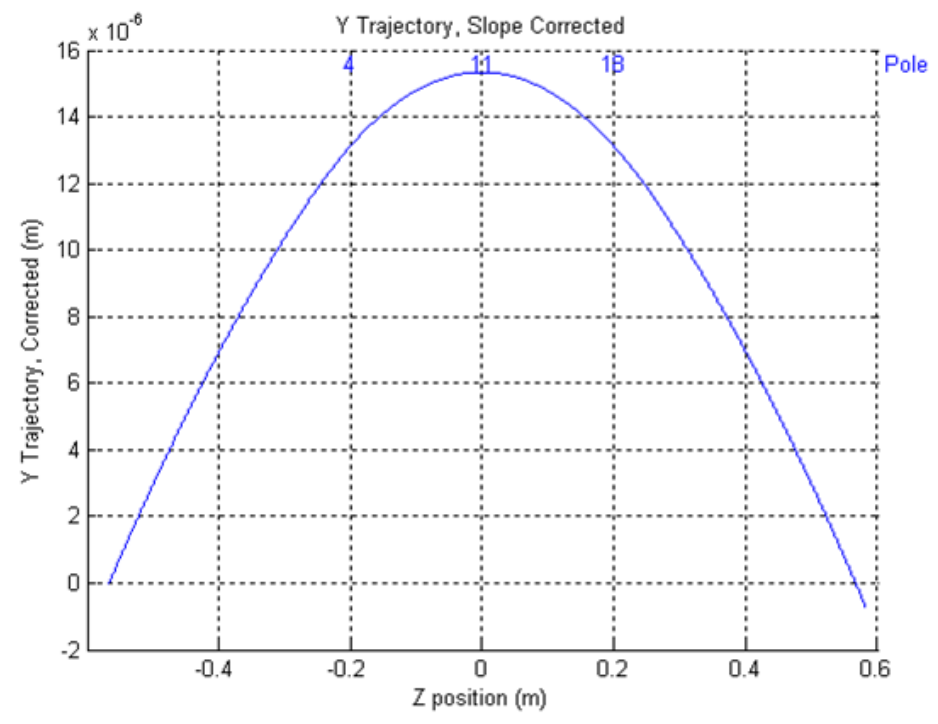

Figure 33: External correctors can be used to remove the average trajectory slope in the undulator with the $0.5 \mathrm{G}$ horizontal field. The resulting vertical trajectory is shown. 


\section{Conclusion}

The construction of the laser heater undulator must include adjustments to the block positions in order to tune the undulator. Both translations toward and away from the gap and rotations about the center of the block face near the gap are necessary for tuning. The range of motion and the required resolution were determined in this note. The sensitivities to block motions of the angle changes for the vertical and horizontal trajectories was determined. The sensitivity of the phase change to block motion was determined. This information will be used in tuning the undulator. It was determined that nearby dipole magnets do not need to be present during tuning. Differences in the Earth's field between the tuning laboratory and the tunnel will require corrector magnets to bring the beam trajectories within tolerance.

\section{Acknowledgements}

Many thanks to Ross Schlueter, Soren Prestemon, Steve Marks, Gordon Bowden, Yurii Levashov, and Richard F. Boyce for valuable discussions. 